\title{
Exact propagation of open quantum systems in a system-reservoir context
}

\author{
JÜrgen. T. Stockburger ${ }^{1}$ \\ ' 1 Institute for Complex Quantum Systems, Ulm University - Albert-Einstein-Allee 11, 89081 Ulm
}

PACS 03.65.Yz - Decoherence; open systems; quantum statistical methods

PACS 05.40.-a - Fluctuation phenomena, random processes, noise, and Brownian motion

PACS 05.70.Ln - Nonequilibrium and irreversible thermodynamics

\begin{abstract}
A stochastic representation of the dynamics of open quantum systems, suitable for nonperturbative system-reservoir interaction, non-Markovian effects and arbitrarily driven systems is presented. It includes the case of driving on timescales comparable to or shorter than the reservoir correlation time, a notoriously difficult but relevant case in the context of quantum information processing and quantum thermodynamics. A previous stochastic approach is re-formulated for the case of finite reservoir correlation and response times, resulting in a numerical simulation strategy exceeding previous ones by orders of magnitude in efficiency. Although the approach is based on a memory formalism, the dynamical equations propagated in the simulations are time-local. This leaves a wide range of choices in selecting the system to be studied and the numerical method used for propagation. For a series of tests, the dynamics of the spin-boson system is computed in various settings including strong external driving and Landau-Zener transitions.
\end{abstract}

Introduction. - The reduced density matrix of an open quantum system is the fundamental mathematical object characterizing the system's state and dynamics. It is defined through a partial trace operation on the density matrix of a larger system, often referred to as "tra' cing out the environment". Finding a suitable description of dynamics for a state which is thus, by definition, an incomplete description of physical reality becomes a nontrivial task, for which a number of techniques, most of ' them approximate, have been developed.

Quantum master equations have been used successfully , where the influence of an environment on the dynamics can be characterized as perturbative and Markovian. Master equations of Lindblad form [1,2] are often preferred since they generate completely positive maps.

Lindblad terms not only reflect properties of the environment and the system-environment interaction, but also the dynamics and level structure of the system, to the degree it can be resolved within time intervals of the order of the correlation time of the environment. In the typical case of an equilibrated environment with thermal energy $k_{\mathrm{B}} T$ lower than the system's level spacing, the dependence of Lindblad operators on the specific properties of the system Hamiltonian is crucial: The thermal timescale $\hbar \beta$ is long enough to differentiate between the energy levels of the system, making the approach difficult to apply to complex or driven systems. This applies in particular when driving is not adiabatic on the timescale of reservoir fluctuations [3]. When driving parameters are chosen such that the level structure of the system changes appreciably over time intervals of width $\hbar \beta$ (thermal timescale), novel effects appear 4] which are not within the scope of standard master equations.

Sometimes ad-hoc combinations of Hamiltonian and Lindbladian terms are used to define a dissipative system. While this approach is guaranteed to result in a completely positive channel, there are important cases where it is impossible to reconcile with the full coupled dynamics of the system coupled to a thermal reservoir [5, 6].

Non-perturbative approaches to open-system dynamics include formally exact dissipative path integrals [7, 8, as well as renormalization group methods 911, which provide a computational approach suited to shed light on quantum phase transitions.

Dissipative path integrals contain a non-local action term [7, which makes it difficult to find a fully equivalent equation of motion for the reduced density matrix. Several strategies have been employed, among them the timediscrete propagation of a multidimensional tensor state [12, equations of motion for a hierarchy of auxiliary dens- 
ity matrices [13], and mapping environmental fluctuations and response on stochastic processes. Several stochastic approaches are known, among them the non-Markovian extension of quantum state diffusion [14, stochastic decoupling of system and reservoir 15,16] and the stochastic unravelling of influence functionals [17, 18. The latter approach is applicable in the generic case of linear dissipation with arbitrary spectral characteristics. In the following, a variant of this approach will be developed which shares features with existing finite-memory approaches to reduced dynamics 19]20 but leads to equations of motion which are time-local and allow arbitrary time-dependent Hamiltonians.

The finite-memory stochastic propagation (FMSP) variant of the stochastic approach presented here shows improvements in efficiency reducing the required computational resources by orders of magnitude. In particular, the revised method is now suitable to fully explore equilibration processes, non-equilibrium steady states and the long-time limit of cyclic processes.

Stochastic Liouville-von Neumann equation. The path integral representation [7 of an open quantum system describes the effects of coupling to an environment in terms of an influence functional, a functional of a pair of forward and backward paths which depends on properties of the system-reservoir coupling, the reservoir dynamics and the initial state of the reservoir. It does not depend on the system's intrinsic (or forced) dynamics in any way, however, it is not a time-local functional. It cannot be transformed into a deterministic equation of motion for the reduced density matrix unless quite restrictive approximations are made or a significant number of auxiliary dynamical states are included [12,13.

In the case of linear dissipation, i.e., Gaussian free fluctuations of the reservoir, the influence functional itself is also a Gaussian functional. Here we consider a systemreservoir interaction $H_{\mathrm{I}}=-q \cdot B$, where $q$ is a system coordinate and $B$ is typically a force depending on many reservoir coordinates, with an obvious generalization to arbitrary sums of separable terms.

The resulting Feynman-Vernon influence functional 7 is completely characterized by the complex-valued correlation function

$$
L\left(t-t^{\prime}\right)=\frac{1}{Z} \operatorname{tr}\left(\exp \left(-\beta H_{\mathrm{R}}\right) B(t) B\left(t^{\prime}\right)\right)
$$

of the free reservoir fluctuations. The function $L\left(t-t^{\prime}\right)$ describes both fluctuations (real part) and dynamical response (back action, imaginary part).

The mathematical structure of a Feynman-Vernon influence functional is closely related to generating functionals of stochastic processes governed by classical probability. This allows a stochastic re-formulation of open-system dynamics in terms of the stochastic Liouville-von Neumann equation 17, 18

$$
i \hbar \frac{d}{d t} \rho=\mathcal{L} \rho=\left[H_{\mathrm{S}}, \rho\right]-\xi[q, \rho]-\frac{\hbar \nu}{2}[q, \rho]_{+}
$$

With its time evolution governed by two correlated stochastic process $\xi(t)$ and $\nu(t)$, the dynamical state $\rho$ itself becomes a stochastic variable; the physical reduced density matrix

$$
\bar{\rho}(t)=\langle\rho(t)\rangle
$$

is obtained as the expectation value of samples $\rho(t)$. Note that angle brackets $\langle\cdot\rangle$ refer to stochastic averages throughout this paper, no trace operation is implied.

For eq. (2) to match the original system-reservoir model, the following conditions are sufficient [18]:

$$
\begin{aligned}
\left\langle\xi(t) \xi\left(t^{\prime}\right)\right\rangle & =\operatorname{Re} L\left(t-t^{\prime}\right) \\
\left\langle\xi(t) \nu\left(t^{\prime}\right)\right\rangle & =(2 i / \hbar) \Theta\left(t-t^{\prime}\right) \operatorname{Im} L\left(t-t^{\prime}\right)+i \mu \delta\left(t-t^{\prime}\right) \\
& =-i \chi\left(t-t^{\prime}\right)+i \delta\left(t-t^{\prime}\right) \int_{0}^{\infty} d \tau \chi(\tau) \delta\left(t-t^{\prime}\right) \\
\left\langle\nu(t) \nu\left(t^{\prime}\right)\right\rangle & =0
\end{aligned}
$$

These conditions can be fulfilled (and corresponding noise samples generated using the fast Fourier transform method) provided that $\xi(t)$ and $\nu(t)$ are allowed to take complex values. The mathematical result (2)-(4) is physically counter-intuitive on the level of individual samples: There is no response or damping term in eq. (2); the dynamic response function $\chi\left(t-t^{\prime}\right)$ emerges only after averaging over noise realizations.

The preceding equations are universally valid for arbitrary dissipation strength and spectral characteristics of the reservoir fluctuations. Unlike Lindblad terms, the stochastic terms in eq. (2) do not depend on $H_{\mathrm{S}}$ in any way; it is perfectly legitimate to modify the Hamiltonian $H_{\mathrm{S}}$ in (2) while keeping the stochastic terms unchanged. In particular, the stochastic Liouville-von Neumann equation can accommodate external driving with arbitrary time dependence, which would break the assumptions underlying the standard derivation of commonly used master equations.

Efficient simulation methods based on slight modifications of eq. (2) have been developed for semiclassical dynamics 21 and for continuous degrees of freedom with ohmic friction 22. In the general case, however, the numerical cost of averaging eq. (2) over explicitly drawn samples of $\xi$ and $\nu$ can be prohibitive. Eq. (2) contains $\xi$ and $\nu$ as multiplicative noise, leading to asymptotic longtime behaviour similar to geometric Brownian motion, the prototypical example of multiplicative noise. Empirical data indicate that the second moment of $\|\rho\|$ (Frobenius norm) exists for arbitrary $t$, but grows exponentially in the asymptotic regime of large $t$. While the method has been found valuable as a computational tool for transient phenomena, even in a system as complicated as the FMO bacteriochlorophyll complex 23, its numerical cost in the long-time limit grows exponentially. The sequel illustrates a refined approach which overcomes this problem.

Finite-memory stochastic propagation. - A frequent situation in the dynamics of an open quantum sys- 
tem interacting with a reservoir is the following: The reservoir correlations have a finite correlation time, yet this time is not short enough to allow a Markovian approximation. Hence, whenever $L(\tau)$ is effectively a function with finite support, modified simulation strategies based on eq. (2) can be found with much more benign requirements of computational resources.

Forming the expectation value $\bar{\rho}=\langle\rho\rangle=\mathcal{P} \rho$ can be viewed as the definition of a projection operation $\mathcal{P}$, with complement $\mathcal{Q}=\mathbb{1}-\mathcal{P}$. Eqs. (2) and (3) are thus related to a Nakajima-Zwanzig equation

$$
\frac{d}{d t} \bar{\rho}=\mathcal{P} \mathcal{L} \bar{\rho}+\mathcal{P} \mathcal{L} \int_{t^{*}}^{t} d t^{\prime} \exp _{>}\left(\mathcal{Q L}\left(t-t^{\prime}\right)\right) \mathcal{Q L} \bar{\rho}\left(t^{\prime}\right),
$$

which is a formal equation of motion for the relevant part $\bar{\rho}=\mathcal{P} \rho$, with $\exp _{>}$denoting a time-ordered exponential.

With $t^{*}=0$ and the initial condition $\mathcal{Q} \rho(0)=0$, this equation is exactly equivalent to eqs. (2) and (3). Now it is important to note that the lower integration boundary can be raised to $t-\tau_{\text {mem }}$ without incurring noticeable errors in the case of finite memory time $\tau_{\text {mem }}$ of the environmental effects.

With $\mathcal{P}$ in the original meaning of "tracing out the environment", time-discrete versions of eq. (5D) with finite memory time have recently been discussed [19, 20, with a focus on explicitly determining the discrete analogue of the memory operator $\mathcal{P} \mathcal{L} \exp _{>}\left(\mathcal{Q L}\left(t-t^{\prime}\right)\right) \mathcal{Q} \mathcal{L}$.

The finite-memory case $t^{*} \lesssim t-\tau_{\text {mem }}$ also benefits the stochastic approach, since the "noisy" propagator $\exp _{>}\left(\mathcal{Q L}\left(t-t^{\prime}\right)\right)$ is then applied only to time intervals of length up to $\tau_{\text {mem }}$ rather than $t$ (in the infinite-memory case, $\left.t^{*}=0\right)$.

An efficient simulation algorithm with greatly improved sampling statistics results from the following approach: Instead of computing the integral in (5) for each time step of a numerical solution, it is advantageous to transform eq. (5) back into a system of differential equations for the relevant part $\bar{\rho}=\mathcal{P} \rho$ and the irrelevant part $\breve{\rho}=\mathcal{Q} \rho$. However, multiple instances of $\breve{\rho}$ are needed since different lower bounds of the integral amount to different initial conditions for $\breve{\rho}$.

It is therefore favourable to choose the lower integration boundary as a piecewise constant staircase function $t^{*}(t)$, bounded by $t-\tau_{\text {mem }}$ from above and by $t-\tau_{\text {mem }}-\tau^{*}$ from below. The "staircase timescale" $\tau^{*}$ is an intermediate time scale shorter than $\tau_{\text {mem }}$, to be discussed in further detail below.

One thus arrives at the equivalent set of coupled equations

$$
\begin{aligned}
\dot{\bar{\rho}} & =\mathcal{P} \mathcal{L} \bar{\rho}+\mathcal{P} \mathcal{L} \breve{\rho}_{m(t)} \\
\dot{\rho}_{n} & =\mathcal{Q} \mathcal{L} \bar{\rho}+\mathcal{Q} \mathcal{L} \breve{\rho}_{n}
\end{aligned}
$$

with the initial conditions $\bar{\rho}(0)=\rho_{0}$ at $t=0$ and $\breve{\rho}_{n}\left(n \tau^{*}\right)=0$ at equidistant times $n \tau^{*}$. Equation (6) is a linear deterministic equation, with an inhomgeneous term $\mathcal{P} \mathcal{L} \breve{\rho}_{m(t)}$ containing the covariance $\left\langle\xi \breve{\rho}_{m(t)}\right\rangle$, while eq. (7) is a linear stochastic equation with inhomogeneity $\mathcal{Q} \mathcal{L} \bar{\rho}$ containing products of $\bar{\rho}$ with either noise variable.

The index $m(t)$ is given by $m(t)=\max (0, L(t-$ $\left.\left.\left.\tau_{\text {mem }}\right) / \tau^{*}\right\rfloor\right)$, where the brackets $\lfloor\cdot\rfloor$ denote the floor function. This ensures that the time interval between the initialization of $\breve{\rho}_{m(t)}$ and its first appearance in eq. (6) is longer than $\min \left(t, \tau_{\mathrm{mem}}\right)$.

General notes on implementation. - Formally, eq. (7) suggests an infinite set of equations. However, at any time $t$ those $\rho_{n}$ with $n>t / \tau^{*}$ have not even been assigned their initial values, and all $\rho_{n}$ with $n<$ $\left(t-\tau_{\mathrm{mem}}\right) / \tau^{*}$ can safely be discarded since $m(t) \neq n$ for all future times. The number of "active" instances $\breve{\rho}_{n}$ being propagated at any given time $t$ is finite, it is bounded by $\tau_{\mathrm{mem}} / \tau^{*}+1$.

The major benefit of this procedure lies in the repeated averaging of partial results in the course of propagating eq. (6) as well as in the re-setting of the initial state of $\breve{\rho}$ : As a consequence of this, the growth rates of the variances of $\|\bar{\rho}\|$ and $\|\breve{\rho}\|$ become arbitrarily small when the number of samples is increased, i.e., the number of samples needed for given $t$ no longer grows exponentially.

The timescale $\tau^{*}$ is chosen to roughly satisfy two criteria: The number of active instances $\approx \tau_{\mathrm{mem}} / \tau^{*}$ should not be too large, and the maximum effective memory time $\tau_{\text {mem }}+\tau^{*}$ should not be much larger than $\tau_{\text {mem }}$. The memory time $\tau_{\text {mem }}$ itself is typically chosen as a multiple of the reservoir correlation time to account for higher-order processes.

Any numerical propagation scheme capable of dealing with equations of type (6) and (7) is compatible with this approach; in particular, its time step can be chosen independently of $\tau^{*}$. In the following examples, a simple split-operator technique is used.

Numerical examples. - The spin-boson model 8 , 24 is a well-studied, yet non-trivial model which will be used to test the performance and versatility of FMSP. In the simplest case, the system part of the spin-boson Hamiltonian is a pseudo-spin subject to a field in $x$ direction. The Hamiltonian for system, coupling and bosonic reservoir can be written as

$$
H=-\frac{\hbar \Delta}{2} \sigma_{x}-\sigma_{z} B+\sum_{j} \hbar \omega_{j} a_{j}^{\dagger} a_{j}
$$

where $\sigma_{z}$ takes the role of $q$ in the preceding formalism, and where $B=\sum_{j} \lambda_{j}\left(a_{j}+a_{j}^{\dagger}\right) / 2$. In the case of ohmic friction, the reservoir is characterized by a dimensionless dissipation constant $\mathrm{K}$, the reservoir temperature, and a spectral density

$$
G(\omega)=\pi \sum_{j} \lambda_{j}^{2} \delta\left(\omega-\omega_{j}\right)=2 \pi K \omega f\left(\omega / \omega_{c}\right)
$$

which rises linearly over a wide range of frequencies $\omega$ and vanishes in the ultraviolet limit $\omega \gg \omega_{c}$. The choice of an 


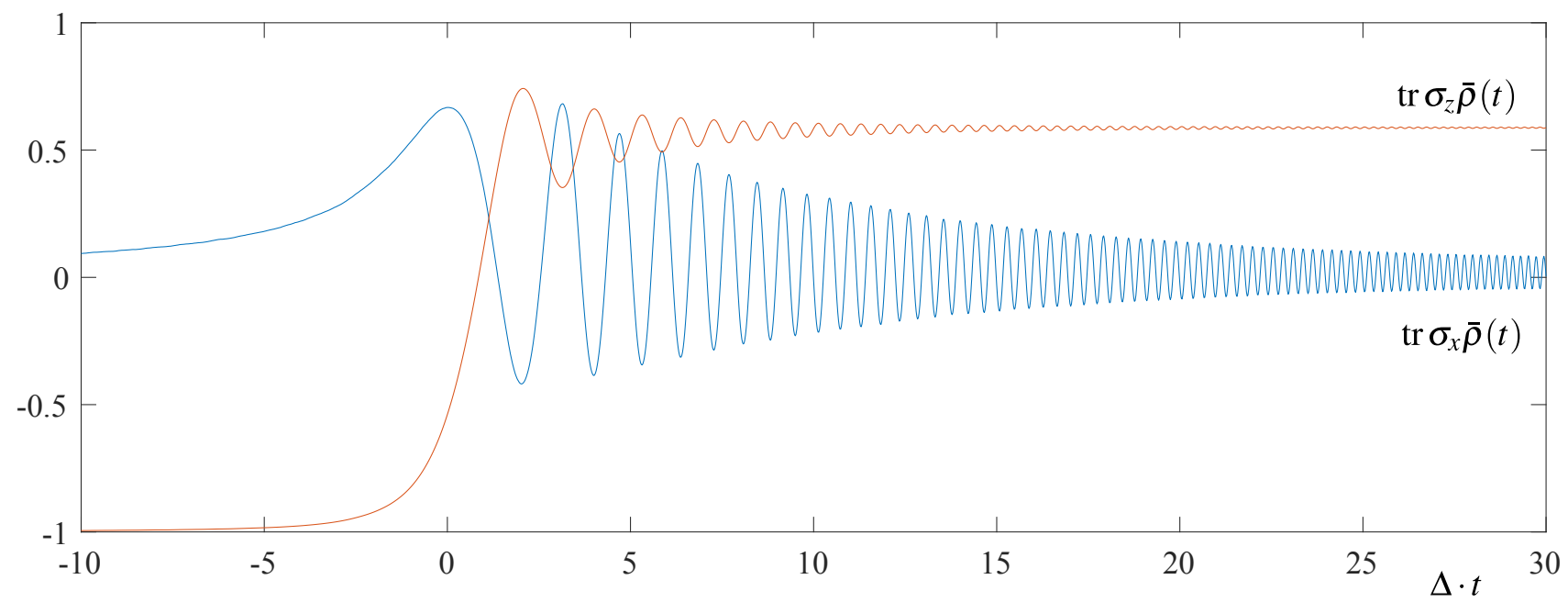

Figure 3: Dissipative Landau-Zener dynamics: Dephasing completes the transition. Parameters are $v=\Delta^{2}, K=0.05, \hbar \beta=2 \Delta^{-1}$ and $\omega_{c}=10 \Delta$. Note that the decay of $\operatorname{tr} \sigma_{x} \bar{\rho}$ is non-exponential.

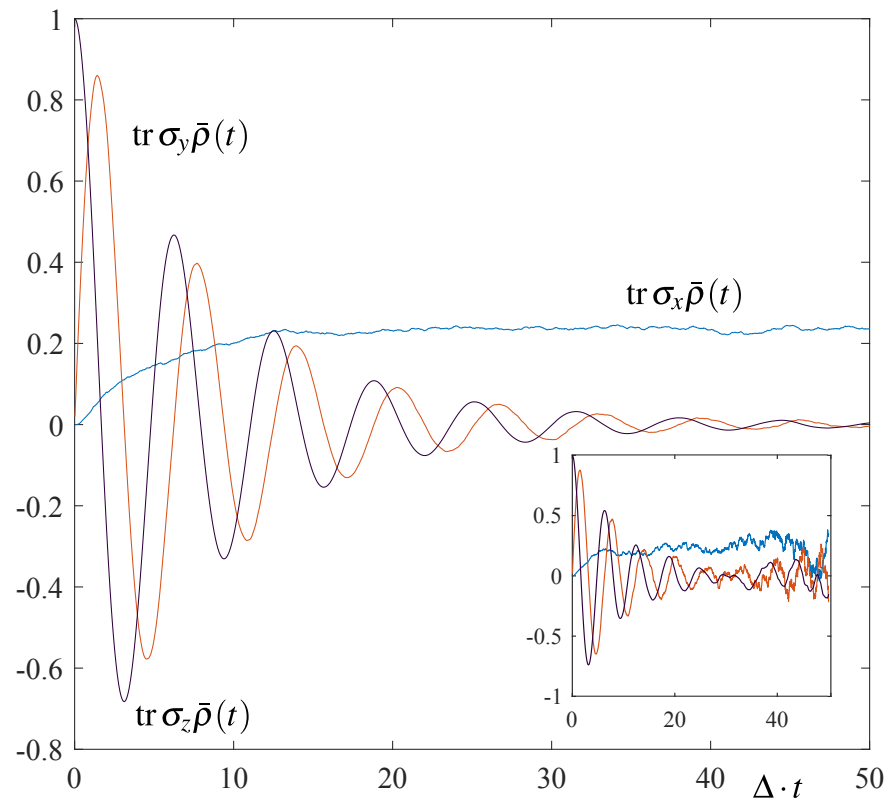

Figure 1: Performance test: Spin-Boson dynamics, ohmic dissipation with dissipation constant 8$] K=0.2$, thermal time (scaled inverse temperature) $\hbar \beta=1 / 2 \Delta^{-1}$ and $\omega_{c}=10 \Delta$. Inset: direct simulation of eq. (2), same parameters. See text for simulation details.

algebraic function $f(x)=\left(1+x^{2}\right)^{-2}$ ensures rapid decay of $\operatorname{Im} L(\tau)$ in the time domain.

Fig. 1 1shows simulation data obtained using FMSP with 500 samples. The system is initially prepared in an eigenstate of $\sigma_{z}$, uncorrelated with the environment. $\operatorname{tr} \sigma_{z} \bar{\rho}$ and $\operatorname{tr} \sigma_{y} \bar{\rho}$ show the expected damped coherent oscillations, while $\operatorname{tr} \sigma_{x} \bar{\rho}$ relaxes towards equilibrium. The inset shows the result of a direct simulation of eq. (2) with an equal number of samples.

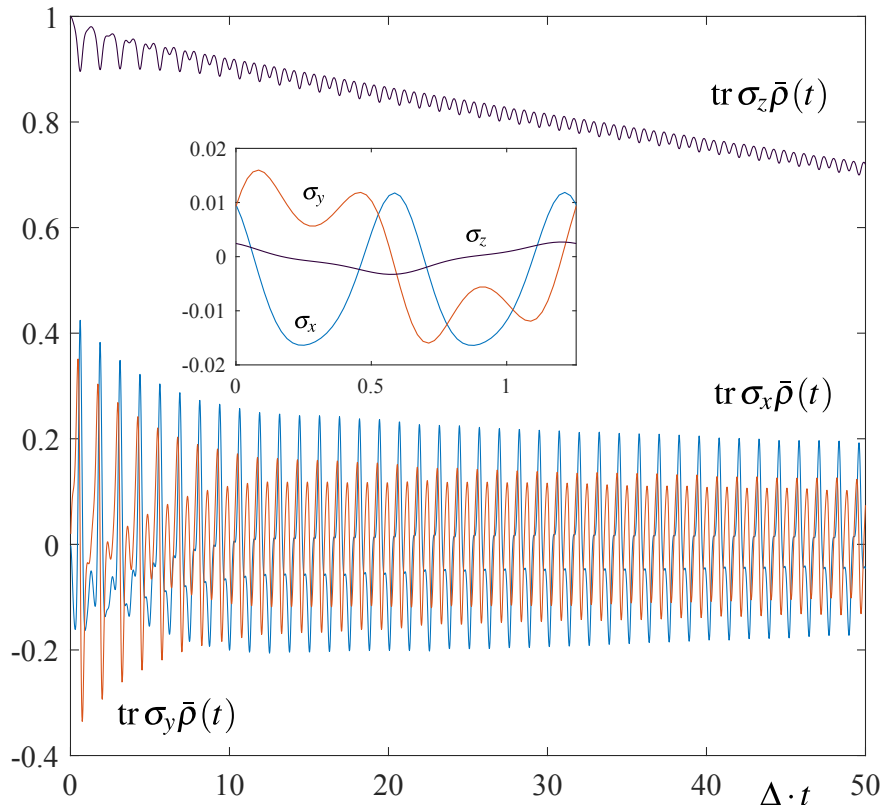

Figure 2: Test case: Spin-Boson dynamics with high-frequency driving, ohmic dissipation with parameters $K=0.02, \hbar \beta=$ $1 / 2 \Delta^{-1}$ and $\omega_{c}=10 \Delta$ and driving with parameters $\epsilon_{1}=12 \Delta$, $\omega_{0}=5 \Delta$. Inset: asymptotic periodic dynamics (scaled time $\Delta \cdot t$ taken modulo driving period).

What is noteworthy about this first test case may not be the physical result per se, but the comparison of the error characteristics of the two approaches. The statistical errors for the observables $\sigma_{j}$ and the Frobenius norm of $\breve{\rho}_{n}$ level out at plateaus at or below an absolute value of $\approx 0.005$. The direct simulation of eq. (2) shows a rapidly deteriorating signal-to-noise ratio at long times (inset); its variance at $t=50 \Delta^{-1}$ is about two orders of magnitude 
larger then for the finite-memory approach. Beyond that time it grows exponentially with a rate roughly equal to the absolute value of the dissipative decay rate.

We now turn to strongly driven spin-boson dynamics, fig. 2, characterized through the Hamiltonian

$$
H=-\frac{\hbar \Delta}{2} \sigma_{x}+\frac{\hbar \epsilon(t)}{2} \sigma_{z}-\sigma_{z} B+\sum_{j} \hbar \omega_{j} a_{j}^{\dagger} a_{j}
$$

with $\epsilon(t)=\epsilon_{1} \cos \left(\omega_{0} t\right), \epsilon_{1}=12 \Delta$ and $\omega_{0}=5 \Delta$. Apart from rapid oscillations, the dynamics shows a dramatic slow-down of relaxation and dephasing, which is expected, since the driving parameters are near the regime of coherent destruction of tunnelling [25, 26]. The inset of fig. 2 shows the asymptotic periodic dynamics, determined from a separate run with symmetric initial condition, extending up to $\Delta \cdot t=2 \cdot 10^{2}$.

The stochastic construction (4) being completely independent of the system Hamiltonian $H_{\mathrm{S}}$, all simulation parameters relating to the dissipation mechanism (coupling strength, noise spectra) are exactly the same as in fig. 1. except that the number of samples has been increased by a factor of four to allow resolution of the finer highfrequency features.

As a model case of non-periodic driving, a Landau-Zener transition is considered next, i.e., driving of the form of a linear sweep $\epsilon(t)=v t$. The dissipative Landau-Zener problem has a known solution for zero temperature 27. and has been studied numerically, using a related stochastic method adapted particularly to ohmic dissipation [28. With a sweep speed $v=\Delta^{2}$, fig. [3 shows both the change in population of $\sigma_{z}$ eigenstates and the gradual loss of coherence (decay of $\operatorname{tr} \sigma_{x} \bar{\rho}$ ) which finalizes the transition. The simulation is based on 30,000 noise samples and covers the interval $\Delta \cdot t \in[-30,30]$. The decay of coherence is somewhat slower than exponential; this is to be expected since the decay mechanism itself is affected by driving. A simulation over the entire interval covered here would have been infeasible using direct sampling of eq. (2).

The thermal timescale of a reservoir is often orders of magnitude larger than its timescale of dynamic response; in the case of an ohmic reservoir these would be $\hbar \beta$ and $1 / \omega_{c}$. It is possible 18 to split the noise $\xi(t)$ into two independent parts, $\xi(t)=\xi^{(s)}+\xi^{(l)}$, where $\xi^{(s)}$ is complexvalued with a short correlation time $1 / \omega_{c}$, and $\xi^{(l)}$ is realvalued with correlation time $\hbar \beta$. The probability space of the noise functions is thus a product of two independent spaces: one with long-range, real-valued noise $\xi^{(l)}$ and one with short-range, complex-valued noise $\left(\xi^{(s)}, \nu\right)$. Applying finite-memory propagation to the short-range part, while directly sampling the long-range part, becomes attractive when stronger dissipation and lower temperatures are to be considered. Simulation results obtained with this approach are shown in fig. 4. It is evident by visual inspection that the numerical error remains roughly constant after the system has equilibrated. This variant of FMSP can therefore be used to gather thermodynamic inform-

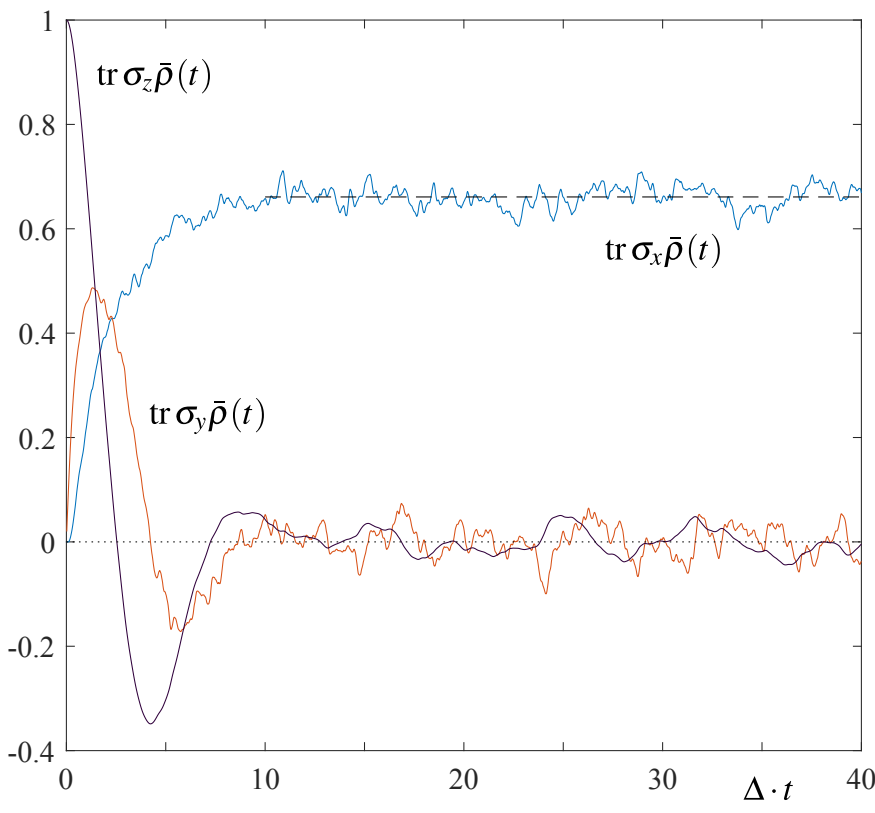

Figure 4: Spin-Boson dynamics with moderate ohmic dissipation, $K=0.2$, and a thermal time $\hbar \beta=5 \Delta^{-1}$. Both time evolution and stationary state differ significantly from predictions of the Born-Markov approximation. The dashed line indicates the equilibrium value of $\operatorname{tr} \sigma_{x} \bar{\rho} \approx 0.66$ estimated from a time average in the interval $\Delta \cdot t \in[10,40]$.

ation from dynamical simulations. Stationary states may be interpreted as equilibrium states in the case of nonperturbative system-reservoir interactions, or, in the case of more than one reservoir, non-equilibrium steady states. Taking time averages over the stationary part of the dynamics, indicated by the dashed line, further reduced statistical errors.

The expectation values $\operatorname{tr} \sigma_{j} \bar{\rho}$ provide a complete parameterization of the reduced density matrix of the two-state system. Due to symmetry, only $\operatorname{tr} \sigma_{x} \bar{\rho}$ is non-zero in the stationary state. Its numerical value leads to quantitative thermodynamic data, e.g., the entropy $S \approx 0.455 k_{\mathrm{B}}$, an order of magnitude larger than the entropy $S \approx 0.0402 k_{\mathrm{B}}$ in the weak-coupling limit, where the occupation of the upper level is less than 2 percent. This strong discrepancy can easily be understood in a two-state model: For stronger coupling, there is a competition between environmentinduced superselection 29] and local equilibration. Decreasing coherence between eigenstates of $\sigma_{z}$ through decoherence automatically leads to a decrease of the population difference of $\sigma_{x}$ eigenstates in the two-state system. The effect observed here is also consistent with a full thermodynamic analysis of the spin-boson model 8 .

Discussion. - The stochastic Liouville-von Neumann equation in its original form (2), which is a universally valid, non-perturbative representation of open-system dynamics becomes computationally expensive in the limit of long times. Empirically, one finds an exponential growth 
$N \approx \exp (\Gamma t)$ of the sample number, where $\Gamma$ is a rate of the same order as the physical relaxation and decoherence rates. This problem is solved using the FMSP method.

Assuming a finite memory time $\tau_{\text {mem }}$ of the dissipative mechanism, the finite-memory stochastic propagation given by eqs. (6) and (7) provides an alternative formulation. Here the most important dimensionless quantity determining the required number of samples is no longer $\approx \Gamma t$, but $\Gamma \tau_{\text {mem. }}$. The algorithmic complexity is now no longer exponential, but linear in time $t$.

The revised method's gain in computational efficiency is typically several orders of magnitude; it is an exponential factor in the limit of long simulation times. It performs exceedingly well in the regime of weak to moderate coupling, but also covers non-perturbative settings with manageable requirements for computational resources.

No strict separation of timescales is assumed for $\tau_{\text {mem }}$, hence non-Markovian effects are within the scope of the method.

The FMSP method is applicable whenever an opensystem problem is posed in terms of a system-reservoir coupling and spectral reservoir characteristics and a nonperturbative approach is preferred over the standard BornMarkov-rotating-wave approximation.

The capacity to include arbitrary driving, including sudden unitary transformations, in the presence of systemreservoir correlations also makes the method a candidate for simulations in the field of multidimensional spectroscopy, where the method of hierarchic auxiliary density matrices is established 30. A combination of hierarchic and stochastic methods 31 may be of benefit here.

In its present form, FMSP offers little benefit in the case of a sluggish bath. Related work on a different type of projector, which sets the off-diagonal elements of $\bar{\rho}$ to zero in addition to the stochastic average, is currently under way. This appears to be suitable when strong reservoir fluctuations lead to short decoherence times. The sub-ohmic case of the spin-boson model [11,32, 33 might be a test case for this variant. Evaluating eq. (5) with this type of projector also establishes links to the diagrammatic NICA expansions of path integrals [34,35].

The combined features of long-time propagation and arbitrary driving are welcome for simulation tasks in the emerging field of quantum engineering, in particular, the simulation of quantum heat engines. For its simplified SLED form 22, the compatibility of the stochastic approach with optimal control has already been proven in a thermodynamic context [4.

The stochastic approach can also describe fluctuations in the heat transfer to a thermal reservoir [36] and can therefore be considered a valuable tool when studying the link between dynamics and novel concepts [37] in the thermodynamics in the quantum regime.

$$
* * *
$$

Stimulating conversations with Joachim Ankerhold and
Michael Wiedmann are gratefully acknowledged. This work was supported by Deutsche Forschungsgemeinschaft through grant AN336/6-1.

References

[1] Alicki R. and Lendi K., Quantum Dynamical Semigroups and Applications Vol. 286 of Lecture Notes in Physics (Springer, Berlin) 1987.

[2] Breuer H.-P. and Petruccione F., The theory of open quantum systems (Oxford University Press) 2002.

[3] Alicki R., Lidar D. A. and Zanardi P., Phys. Rev. A, 73 (2006) 052311.

[4] Schmidt R., Negretti A., Ankerhold J., Calarco T. and Stockburger J. T., Phys. Rev. Lett., 107 (2011) 130404.

[5] Levy A. and Kosloff R., EPL (Europhysics Letters), 107 (2014) 20004.

[6] Stockburger J. T. and Motz T., Thermodynamic deficiencies of some simple Lindblad operators arXiv:1606.04326 (2016).

[7] Feynman R. P. and Vernon F. L., Ann. Phys. (N. Y.), 24 (1963) 118.

[8] Weiss U., Quantum dissipative systems 4th Edition (World Scientific) 2012.

[9] Kehrein S. K. and Mielke A., Annalen der Physik, 509 (1997) 90.

[10] Keil M. and Schoeller H., Phys. Rev. B, 63 (2001) 180302.

[11] Anders F. B., Bulla R. and Vojta M., Physical Review Letters, 98 (2007) 210402.

[12] Makarov D. E. and Makri N., Chem. Phys. Lett., 221 (1994) 482.

[13] Tanimura Y. and Kubo R., J. Phys. Soc. Jpn., 58 (1989) 101.

[14] Diósi L. and Strunz W. T., Phys. Lett. A, 235 (1997) 569.

[15] Shao J., J. Chem. Phys., 120 (2004) 5053.

[16] Lacroix D., Phys. Rev. A, 72 (2005) 013805.

[17] Stockburger J. T. and Grabert H., Phys. Rev. Lett., 88 (2002) 170407.

[18] Stockburger J. T., Chem. Phys., 296 (2004) 159.

[19] Golosov A. A., Friesner R. A. and Pechukas P., J. Chem. Phys., 110 (1999) 138.

[20] Cerrillo J. and CaO J., Phys. Rev. Lett., 112 (2014) 110401.

[21] Koch W., Grossmann F., Stockburger J. T. and Ankerhold J., Phys. Rev. Lett., 100 (2008) 230402.

[22] Stockburger J. T. and Mak C. H., J. Chem. Phys., 110 (1999) 4983.

[23] Imai H., Ohtsuki Y. and Kono H., Chemical Physics, 446 (2015) 134.

[24] Leggett A. J., Chakravarty S., Dorsey A. T., Fisher M. P. A., Garg A. and Zwerger W., Rev. Mod. Phys., 59 (1987) 1 ibid. 67, 725 (1995) (erratum).

[25] Grossmann F., Dittrich T., Jung P. and Hänggi P., Phys. Rev. Lett., 67 (1991) 516.

[26] Stockburger J. T., Phys. Rev. E, 59 (1999) R4709.

[27] Wubs M., Saito K., Kohler S., HängGi P. and KaYanuma Y., Phys. Rev. Lett., 97 (2006) 200404. 
[28] Orth P. P., Imambekov A. and Le Hur K., Phys. Rev. A, 82 (2010) 032118.

[29] Zurek W. H., Rev. Mod. Phys., 75 (2003) 715.

[30] Ishizaki A. and Tanimura Y., J. Chem. Phys., 125 (2006) 084501.

[31] Zhou Y., Yan Y. and Shao J., Europhys. Lett., 72 (2005) 334.

[32] Winter A., Rieger H., Vojta M. and Bulla R., Physical Review Letters, 102 (2009) 030601.

[33] Kast D. and Ankerhold J., Physical Review Letters, 110 (2013) 010402.

[34] Egger R., Mak C. H. and Weiss U., Phys. Rev. E, 50 (1994) R655.

[35] Thorwart M., Grifoni M. and Hänggi P., Annals of Physics, 293 (2001) 15.

[36] Schmidt R., Carusela M. F., Pekola J. P., Suomela S. and Ankerhold J., Phys. Rev. B, 91 (2015) 224303.

[37] Brandão F., Horodecki M., NG N., Oppenheim J. and Wehner S., Proceedings of the National Academy of Science, 112 (2015) 3275. 